\title{
Effect of the grooved $\mathrm{SrTiO}_{3}$ bicrystal line on the $\mathrm{YBa}_{2} \mathrm{Cu}_{3} \mathrm{O}_{7}$ grain boundary
}

\author{
Hsiao-Wen $\mathrm{Yu}^{\mathrm{a}, *}$, Ming-Jye Chen ${ }^{\mathrm{a}}$, H.C. Yang ${ }^{\mathrm{a}}$, S.Y. Yang ${ }^{\mathrm{b}}$, H.E. Horng ${ }^{\mathrm{b}}$ \\ a Department of Physics, National Taiwan University, Taipei 106, Taiwan \\ b Department of Physics, National Taiwan Normal University, Taipei 117, Taiwan
}

Received 1 November 1999; received in revised form 25 November 1999; accepted 26 January 2000

\begin{abstract}
Using atomic force microscope (AFM), we have investigated the correlation between the characteristics of grooved $\mathrm{SrTiO}_{3}$ (STO) bicrystal line and the grain-boundary (GB) microstructure of the $\mathrm{YBa}_{2} \mathrm{Cu}_{3} \mathrm{O}_{y}$ (YBCO) film. When the underlying groove becomes deeper and steeper, the density of growth spirals along the boundary line increases and the meandering configuration of GB gradually disappears. From the measured signals of the dc-SQUIDs patterned on the films, we also conclude that the homogeneity of the GB is improved. (C) 2000 Elsevier Science B.V. All rights reserved.
\end{abstract}

PACS: $74.50 .+\mathrm{r}$

Keywords: Meandering grain boundary; Grooved bicrystal line; Microstructure of the grain boundary; SQUID

\section{Introduction}

The superconducting quantum interference devices (SQUIDs) exhibit significantly high sensitivity, which is up to several orders of magnitude higher than the sensitivity of electrical measurement [1]. Since the discovery of high-temperature superconductivity, the SQUID-based instruments are more feasible. Recently, there are encouraging progress in the performance of SQUID made from high- $T_{c}$ superconductor (HTS) thin films. It's commercial application ranges from geophysics, medicine, engineering to NDE investigation. In particular, the

\footnotetext{
* Corresponding author. Tel.: +886-2-23626937 ext. 101; fax: +886-2-23639984.

E-mail address: d3202005@ms.cc.ntu.edu.tw (H.W. Yu).
}

$\mathrm{SrTiO}_{3}$ (STO) bicrystal substrates are commonly used to grow films for their convenience of straightforward fabrication. However, the reproducibility of bicrystal junctions, with a predictable critical current $I_{\mathrm{c}}$ and finite-voltage resistance $R_{n}$, depends on the microstructure of the grain-boundary (GB) interface. It has been pointed out that the grain-boundary junctions (GBJs) are inhomogeneous and composed of parallel weakly and strongly coupled path [2-5]. On the one hand, the spatial nonuniformity could be attributed to the meandering growth mechanism of the film [6-8]. The underlying substrate quality at the fusion boundary, such as defects embedded [9], and the groove formed mainly in the thermal treatment process [10-12], also affect the growth of the over layer film. In those works, scanning probe microscope (SPM) and near-field scanning optical 
microscopy (NSOM) are especially used for their nondestructivity.

To our knowledge, annealing STO bicrystal substrate at high temperature could be employed in the film deposition process or to reduce surface roughness after polishing. This results in a wide range of thermal treatment duration. Thus, the influence of zigzagging groove on the GB depends on the different film preparation conditions. Therefore, it requires a further understanding of the relation between the intrinsic GB structure and the characteristic of the underlying grooved bicrystal line.

In this letter, atomic force microscope (AFM) was used to study the relation between the structural properties of the grooved STO bicrystal substrates and the interface of $\mathrm{YBa}_{2} \mathrm{Cu}_{3} \mathrm{O}_{y}(\mathrm{YBCO}) \mathrm{GBs}$ grown on them. Then, dc-SQUIDs were fabricated on those YBCO films. Because the AFM investigation is nondestructive, the measured SQUID signal can be directly correlated to the boundary microstructure characteristics.

\section{Experimental}

The bicrystal substrates are commercial products and the grooved ones are prepared from the reused bicrystal substrates. All are $2 \times 12^{\circ}$ tilted bicrystals with a common (001) surface of two single-crystal constituents. The thermal heating during deposition, annealing in the cooling process and wet etching with chemicals can lead to the formation of the grooves along the bicrystal line [10-12]. A schematic drawing of the grooved substrate is shown in Fig. 1. After AFM investigations, the YBCO films, about $200 \mathrm{~nm}$ thick, were deposited onto those substrates

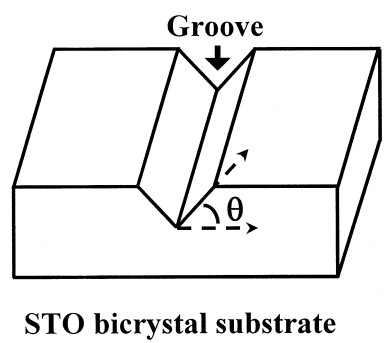

Fig. 1. Schematic illustration of the grooved substrate. $\theta$ is the step angle of the groove. by off-axis sputtering. The as-grown YBCO films have critical temperatures up to $91 \mathrm{~K}$. The rms roughness of the films is about $7 \mathrm{~nm}$. Conditions of thin film preparation have already been published in detail [13]. The dc-SQUIDs were patterned with the standard photolithography and wet etching. Thereafter, $200 \mathrm{~nm}$ thick gold was evaporated onto the YBCO pads for ultrasonic wire bonding.

\section{Results and discussion}

Since a new substrate has been polished to a root-mean-square roughness of 5-10 $\AA$, the bicrystal line could not be identified in the smooth surface topography taken from the AFM. However, the optical microscope could detect the defects embedded in the bicrystal fusion. The optical photograph is shown in Fig. 2, while the inset displays an image at higher magnification. The dark circles in those pictures are the defects embedded. Those defects with a diameter of $\sim 0.2 \mu \mathrm{m}$ spreads arbitrarily over length scales reaching hundreds of microns and the fabricated junctions hardly have no defect, which cause the GB wandering largely [9]. Fig. 3 displays AFM images from different area in the YBCO film grown on the new bicrystal substrate. The surface morphology from a randomly selected area in the film is shown in Fig. 3 (a). The average size of an island is about $250 \mathrm{~nm}$. The meandering GB formed by the coalescence of irregularly shaped islands is shown in Fig. 3(b). In Fig. 3(b), the bicrystal line of the underlying substrate is marked with a white dash line on the figure while the meandering YBCO GB line is marked by black dots. At the boundary, the film thickness is reduced, compared to the area between the growth islands. The thickness depression originates from the underlying grooved substrate formed during the film deposition process [11]. In particular, the magnitude of GB deviation varies along the bicrystal line, reaching $200 \mathrm{~nm}$ in places, and the magnitude of the mean meandering period is about $250 \mathrm{~nm}$. Both are the same as the magnitude of the islands in Fig. 3(a). It confirms that the island-like growth mechanism of the YBCO films is responsible for the meandering phenomenon. That is, the islands nucleate on one side of the bicrystal boundary keep their lateral 


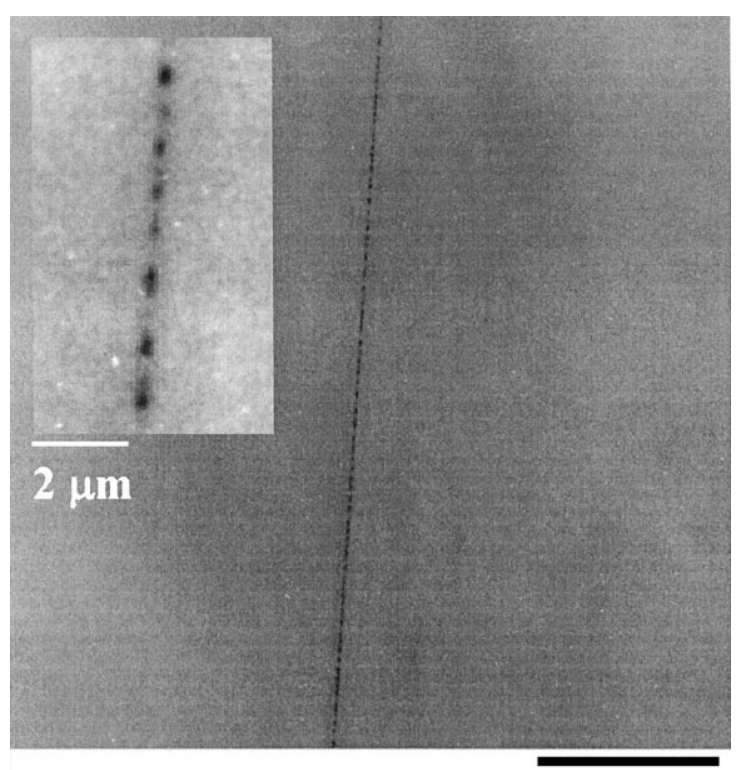

\section{$25 \mu \mathrm{m}$}

Fig. 2. Optical photograph of the bicrystal boundary. It is $100 \times 100$ $\mu \mathrm{m}$ in size. Dark circles spreading along the straight line are the defects embedded. Those defects with a diameter of $\sim 0.2 \mu \mathrm{m}$ is easily identified in the inset at higher magnification.

growth during the deposition process, overgrow across the bicrystal line and coalesce with islands of the other side of the bicrystal. In this way, the meandering GB is formed despite of the poor matching between the film and substrate in the overgrowth area. However, as shown in Fig 3(c), there exists another kind of meandering configuration that sharply deviates from the bicrystal line with a magnitude as much as $0.7 \mu \mathrm{m}$. The significant boundary meandering is caused by the underlying defects in the fusion line [9].

The presence of all the meandering results in a structural inhomogeneity. The fact that the average magnitude of a meandering period is about $250 \mathrm{~nm}$

Fig. 3. Surface morphology taken using AFM from different area in the YBCO film deposited on the new bicrystal substrate. They are $1.5 \times 1.5 \mu \mathrm{m}$ in size. (a) shows the island-like growth mechanism of YBCO film. The meandering YBCO GB is marked by black dots in (b). The sharply meandering GB is displayed in (c), which is caused by the embedded defect. The distance between two white arrows in (c) is about $0.7 \mu \mathrm{m}$. implies that a junction $5 \mu \mathrm{m}$ wide could contain 20 meandering periods. Since there are several facets composed in a meandering period [14], the measured
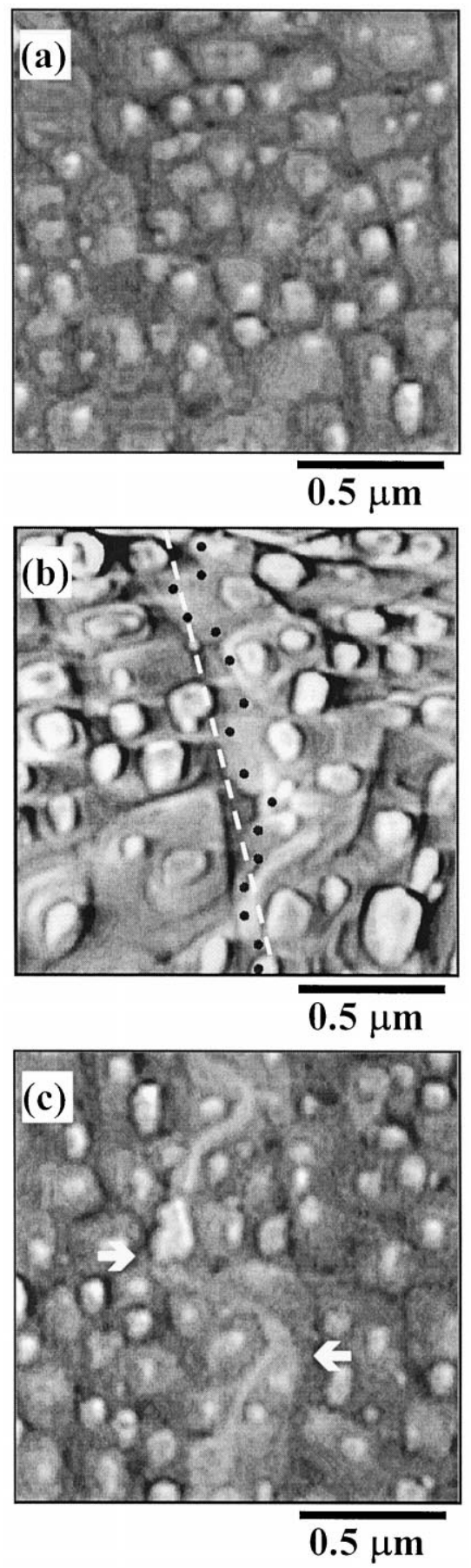
electrical properties will be averaged over those microstructural segments. Besides, as shown in Fig 3(a), (b) and (c), precipitates spreading on the surfaces with size exceeding $100 \mathrm{~nm}$ are easily seen, which have been observed by many groups on their films with the best electrical transport properties $[15,16]$. However, those precipitates on the surface or in the film are not desirable for fabrication of devices.

The images of the prepared grooved substrate 1 and substrate 2 are shown in Fig. 4(a) and (b), respectively. The groove of substrate 2 is deeper and steeper. The average depth $\Delta h$, width $\Delta w$, and step angle $\theta$ of the grooves are listed in Table 1. The GBs of YBCO films grown on the corresponding grooved substrates 1 and 2 are shown in Fig. 5(a) and (b). In Fig. 5(a), the meandering configuration of the interface still exists, but there is a significant thickness depression appearing on the upper portion
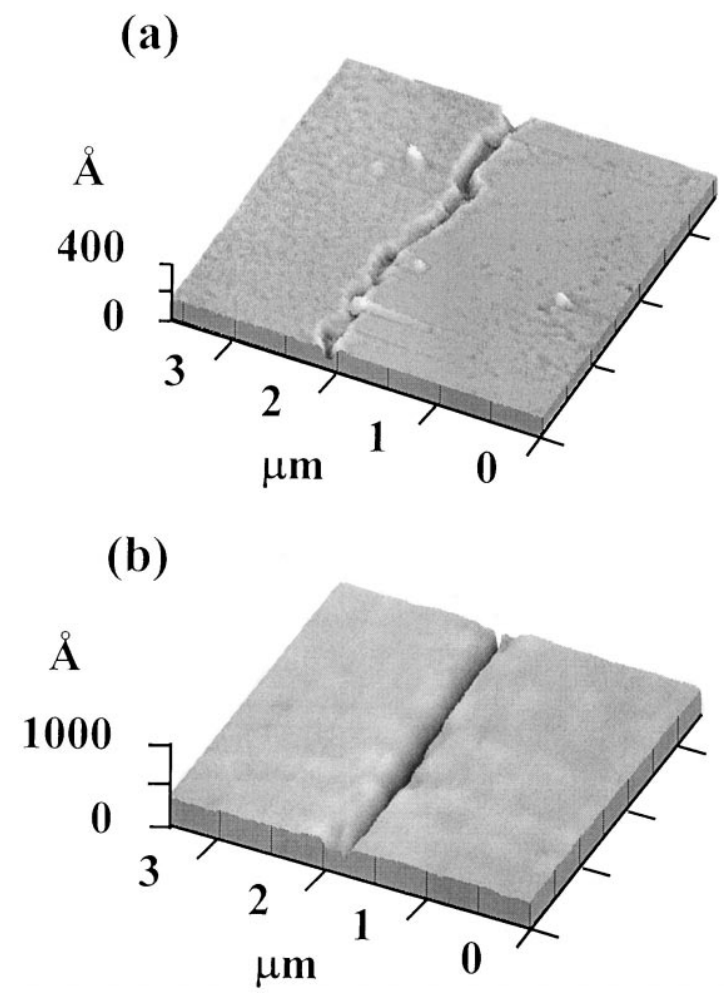

Fig. 4. Topographic images of the prepared grooved substrates with a scan size of $3.5 \times 3.5 \mu \mathrm{m}$. (a) and (b) are the images of grooved substrate 1 and substrate 2 , respectively.
Table 1

Sample data including the average depth $\Delta h$, width $\Delta w$, step angle $\theta$ of the grooved substrates, and the thickness depression $\Delta d$ of the GB for the film deposited on them

\begin{tabular}{lllrl}
\hline & $\Delta h(\mathrm{~nm})$ & $\Delta w(\mu \mathrm{m})$ & $\theta$ & $\Delta d(\mathrm{~nm})$ \\
\hline Substrate 1 & 10 & 0.2 & $7^{\circ}$ & 16 \\
Substrate 2 & 40 & 0.2 & $27^{\circ}$ & 50 \\
\hline
\end{tabular}

and the spiral structure of grain is obvious. In Fig. 5(b), the GB is depressed everywhere and the spirals increase along the bicrystal line, which is correlated to the underlying damaged area. Moreover, the meandering configuration of GB gradually disappears. The most likely explanation of this phenomenon is that the islands nucleate in the vicinity of the boundary hardly keep their lateral growth when they overgrow the groove, which is not favorable for epitaxial growth. The average depression $\Delta d$ of the film on the GB is also listed in Table 1 in comparison with the average depth $\Delta h$ of the groove. These data show that the depth of the grooves is developed during film deposition. For example, the average depth $\Delta h$ of the grooved substrate 1 without the YBCO film is $10 \mathrm{~nm}$, while the average depression $\Delta d$ of the corresponding YBCO GB is $16 \mathrm{~nm}$. This situation also happens to the grooved substrate 2 .

The dc-SQUIDs (SQUID 1, SQUID 2 and SQUID 3), involving $5 \mu \mathrm{m}$ wide junctions, were fabricated on those three films separately. All the resistance vs. temperature $(R-T)$ curves show good superconducting transition with the $T_{\text {c,zero }}$ above $77 \mathrm{~K}$. But the magnetic field dependence of the voltage modulation ( $V-\Phi$ curves) becomes clearer at a lower temperature. Fig. 6(a), (b) and (c) are the $V-\Phi$ curves for SQUID 1, SQUID 2 and SQUID 3 measured within $\pm 40 \mathrm{mG}$. The inset in Fig. 6(a) and (b) displays modulation patterns measured within a larger magnetic field. There are some bizarre characteristics in Fig. 6(a). The shape of the $V-\Phi$ curve is quite complicated, and even the interference figure cannot easily be identified at first sight. Therefore, a circle is used in the right hand side to enclose the interference figure whose period is on the order of the expected magnetic value. In addition, there is a plain region in the middle of the curve, which gives no response to the field variation. We attribute this 
behavior to the specific non-equivalence of the critical current $\left(I_{\mathrm{c}}\right)$ for the two junctions consisting of SQUID 1 [17]. The interference result becomes clear
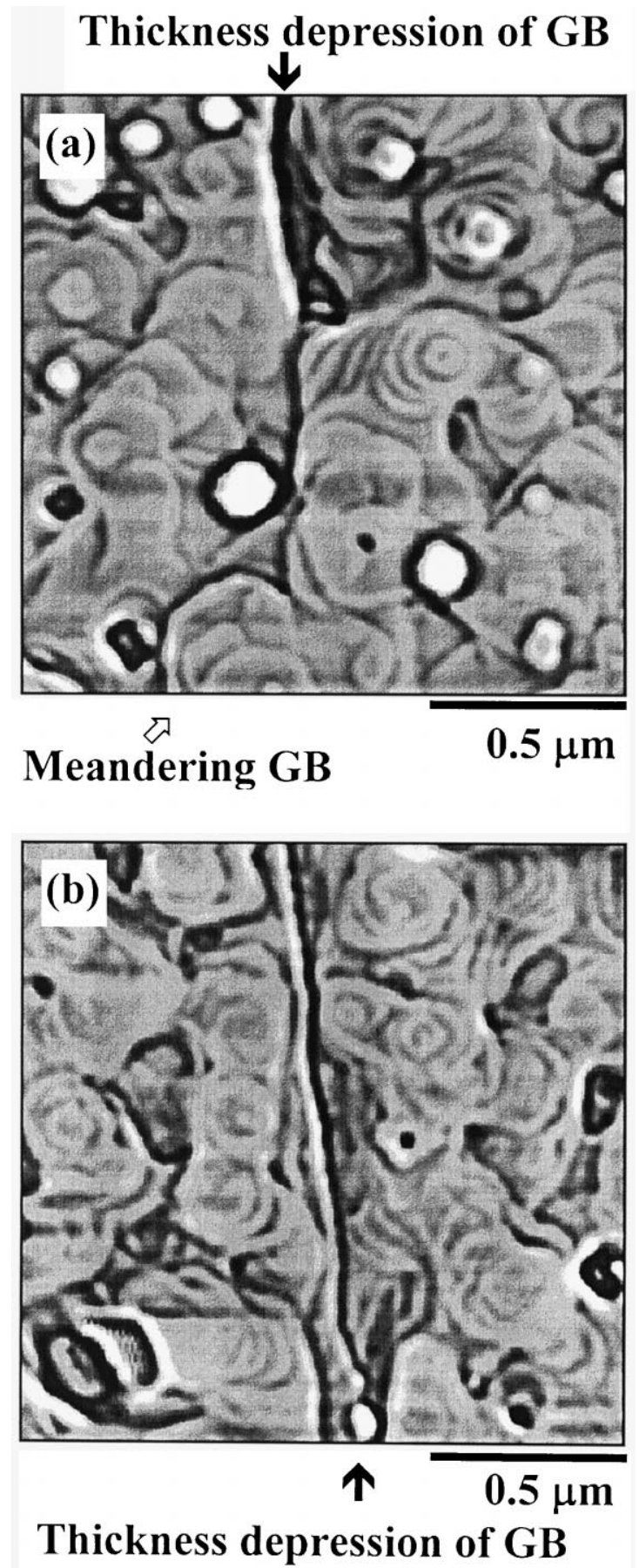

only when the applied field is increased to suppress the tunneling currents and the non-equivalence is less. In the recent NSOM experiment reported by McDaniel et al. [9], the on-chip variation of $I_{c}$ could exceed two to three orders, depending on the number of defects in a junction. This variation is so large that our assumption seems to make sense. Moreover, the period of voltage oscillation in the inset of Fig. 6(a), which is measured within a larger field interval, indicates intrinsic dc-SQUIDs in the junction area with a quite small effective area. In the focused ion beam (FIB) work, this phenomenon is also seen and is related to the existence of microshorts in the junction area [18]. In addition, similar oscillation is observed in the study of YBCO microbridge and is attributed to the presence of weak links in the bridge area [19]. Thus, the measured results of SQUID 1 indicate specific non-uniformity of the GBJs consisting of weak links and microshorts, distributed randomly along the GB. In Fig. 6(b), the plain region reduces and the interference figure becomes more clear, but is still irregular. The voltage modulation in the inset of Fig. 6(b) shows the expected curvature due to the magnetic field sensed by the GBJ, and the periodic oscillation related to the existence of microshorts is absent. In Fig. 6(c), the interference figure behaves quite well and the plain region disappears.

The evolutionary regularity of the $V-\Phi$ curves is attributed to the improvement of the current inhomogeneity along the GB. It also provides direct evidence that the underlying grooved bicrystal line essentially affects the YBCO GB. From the information regarding grooves listed in Table 1 , it is obvious that the step angles are not steep enough that $90^{\circ}$ GBs cannot be formed near the step edges [20]. But on the damaged groove area, the film might show degraded superconducting property allowing the formation of weak links more easily. This is similar to

Fig. 5. Surface morphology of the GB after $200 \mathrm{~nm}$ YBCO film was deposited on the grooved substrates 1 and 2 . They are $1.5 \times 1.5 \mu \mathrm{m}$ in size. The black arrows indicate the thickness depression of GB, while the white arrow indicates the meandering GB. The successive change of the GB microstructure seen from Fig. 3; (a) and (b) show the influence of the underlying grooved substrate. 


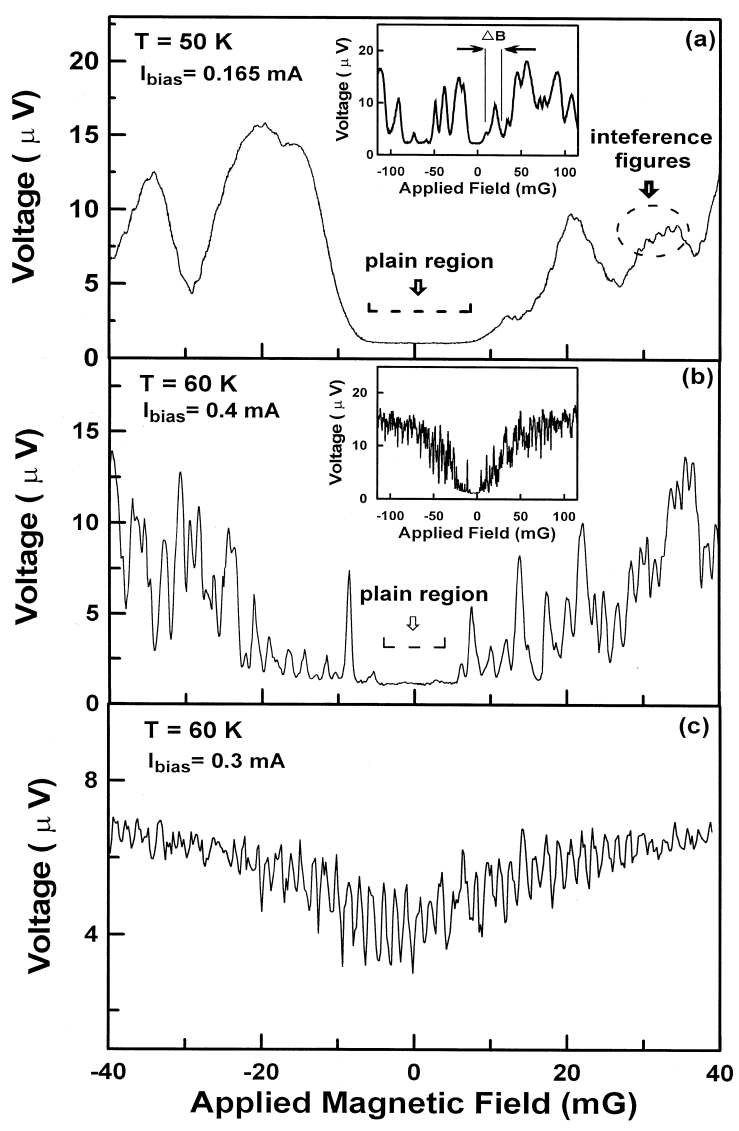

Fig. 6. The evolution of the SQUID signals. (a), (b) and (c) are the $V-\Phi$ curves of SQUID 1, SQUID 2 and SQUID 3 within \pm 40 $\mathrm{mG}$. The bias current $\left(I_{\text {bias }}\right)$ is applied to optimize the voltage modulation depth $(\Delta V)$ for each measurement. The plain region in the middle of the curves is supposed to be correlated to the asymmetry of the $I_{\mathrm{c}}$ between the two junctions. Insets of (a) and (b) show the data measured within $\pm 130 \mathrm{mG}$. The voltage oscillation in the inset of (a) has a modulation period $(\Delta B)$ about $30 \mathrm{mG}$.

the mechanism forming junctions in the FIB technique [21]. Besides, as noted above, the meandering configuration of GB is gradually disappears when the groove becomes steeper and deeper. As a consequence, the structural inhomogeneity of the GB is improved.

It is noticed that SQUID 1 is not working as well as one's expectation. We exclude the suspicion of the junction processing because we have fabricated SQUIDs in the same way on step-edge boundary and they behave well $[22,23]$. The most reasonable expla- nation is that the quality of the bicrystal substrate and YBCO film is not good enough. All the magnitude of the strikingly GB meandering caused by the defects, the GB deviation due to island-growth mechanism and the precipitates on the surface or in the film is on a sub-micrometer scale, which is not desirable for fabrication of devices. Changing the growth condition could vary the quality of the film, however, the reduction of the influence of GB defects is limited because the bicrystal substrate is commercial product and its quality depends on the supplier. Nevertheless, this study concentrates only on the correlation between the grooved bicrystal line and the property of YBCO GB. How to promote the film quality and reduce the influence of GB defects remains a further work to be investigated.

\section{Conclusion}

In conclusion, we have shown the correlation between the grooved bicrystal boundary, the GB microstructure and the $V-\Phi$ behavior of the dcSQUID. To a certain extent, the groove could be a positive factor to improve the non-uniformity of GBJ. In addition, the formation of the grooves should be taken into account as an important parameter in the device fabrication process. Moreover, since the groove is formed mainly in the thermal treatment process, one can control the influence of groove on the GB in the film preparation condition and also optimize the GBJ characteristics. Therefore, the reproducibility of the GBJ could be improved in the future works.

\section{Acknowledgements}

Financial support from the National Science Council of the ROC under grants NSC87-2112-M002-002, NSC87-2112-M-003-001 and NSC872112-M-003-008 is gratefully acknowledged.

\section{References}

[1] R. Meservey, J. Appl. Phys. 39 (1968) 2598.

[2] E.A. Early, R.L. Steiner, A.F. Clark, Phys. Rev. B 53 (1994) 9409. 
[3] M.B. Field, D.C. Larbalestier, A. Parikh, K. Salama, Physica C 280 (1997) 221.

[4] D.K. Lathrop, B.H. Moeckly, S.E. Russek, R.A. Buhrman, Appl. Phys. Lett. 58 (1991) 1095.

[5] O.M. Froehlich, H. Schulze, A. Beck, B. Mayer, L. Alff, R. Gross, R.P. Huebener, Appl. Phys. Lett. 66 (1995) 2289.

[6] X.F. Zhang, D.J. Miller, J. Talvacchio, J. Mater. Res. 11 (1996) 2440.

[7] H. Hilgenkamp, J. Mannhart, B. Mayer, Phys. Rev. B 53 (1996) 4586.

[8] D.J. Miller, T.A. Robers, J.H. Kang, J. Talvacchio, D.B. Buchholz, R.P.H. Chang, Appl. Phys. Lett. 66 (1995) 2561.

[9] E.B. McDaniel, S.C. Gausepohl, C.-T. Li, J.W.P. Mark Lee, R.A. Hsu, C.B. Rao, Appl. Phys. Lett. 70 (1997) 1882.

[10] Q.D. Jiang, X.Q. Pan, J. Zegenhagen, Phys. Rev. B 56 (1997) 6947.

[11] Q.D. Jiang, Z.J. Hung, A. Brazdeikis, M. Dezaneti, C.L. Chen, P. Jin, C.W. Chu, Appl. Phys. Lett. 72 (1998) 3365.

[12] H.-W. Yu, M.-J. Chen, H.-C. Yang, S.Y. Yang, H.E. Horng, IEEE Trans. Appl. Supercond. 9 (1999) 3101.

[13] L.M. Wang, H.W. Yu, H.C. Yang, H.E. Horng, Physica C 256 (1996) 57.
[14] C. Træholt, J.G. Wen, H.W. Zandbergen, Y. Shen, J.W.M. Hilgenkamp, Physica C 230 (1994) 425.

[15] Yu.N. Drozdov, S.V. Gaponov, S.A. Gusev, E.B. Kluenkov, Yu.N. Nozdrin, V.V. Talanov, A.K. Vorobiev, IEEE Trans. Appl. Supercond. 7 (1997) 1642.

[16] H.Z. Chen, T.C. Chow, M.T. Hong, T.L. Lin, Y.F. Cheng, Y.C. Chen, H. Chou, Physica C 274 (1997) 24.

[17] C. Camerlingo, M.P. Janawadkar, M. Russo, G. Paterno, IEEE Trans. Magn. MAG 23 (1987) 696.

[18] Y. Soutome, Y. Okabe, IEEE Trans. Appl. Supercond. 7 (1997) 2311.

[19] S.K. Mishra, L.C. Pathak, V. Rao, D. Bhattacharya, K.L. Chopra, J. Supercond. 9 (1996) 211.

[20] C.L. Jia, B. Kabius, K. Urban, K. Herrman, G.J. Cui, J. Schubert, W. Zander, A.I. Braginski, C. Heiden, Physica C 175 (1991) 545.

[21] Ch. Neumann, K. Yamaguchi, K. Hayashi, Y. Suzuki, S. Enomoto, S. Tanaka, Physica C 210 (1993) 138.

[22] H.E. Horng, J.H. Lu, J.M. Wu, S.Y. Yang, H.C. Yang, J.D. Chen, IEEE Trans. Appl. Supercond. 7 (1997) 3690.

[23] S.Y. Yang, C.H. Chen, H.E Horng, W.L. Lee, H.C Yang, IEEE Trans. Appl. Supercond. 9 (1999) 3121. 